\title{
A facile method to compare EFTEM maps obtained from materials changing composition over time
}

Running title: Comparing elemental maps from in situ EFTEM

Alberto Casu*, Alessandro Genovese, Cristiano Di Benedetto, Sergio Lentijo-Mozo, Elisa Sogne, Efisio Zuddas, and Andrea Falqui*

King Abdullah University of Science and Technology (KAUST) - Biological and Environmental Sciences and Engineering Division - Thuwal 23955-6900, Kingdom of Saudi Arabia

${ }^{*}$ Correspondence to: A. Casu, A. Falqui: King Abdullah University for Science and Technology (KAUST), Biological and Environmental Sciences and Engineering Division, Thuwal 23955-6900, Kingdom of Saudi Arabia. E-mail: alberto.casu@kaust.edu.sa; andrea.falqui@kaust.edu.sa

Key words: in situ EFTEM, elemental maps, digital image treatment

\begin{abstract}
Energy Filtered Transmission Electron Microscopy (EFTEM) is an analytical tool that has been successfully and widely employed in the last two decades for obtaining fast elemental maps in TEM mode. Several studies and efforts have been addressed to investigate limitations and advantages of such technique, as well as to improve the spatial resolution of compositional maps. Usually, EFTEM maps undergo post-acquisition treatments by changing brightness and contrast levels, either via dedicated software or via human elaboration, in order to maximize their signal to noise ratio and render them as visible as possible. However, elemental maps forming a single set of EFTEM images are usually subjected to independent map-by-map image treatment. This post-acquisition step becomes crucial when analyzing materials that change composition over time as a consequence of an external stimulus, because the map-by-map approach doesn't take into account how the chemical features of the imaged materials actually progress, in particular when the investigated elements exhibit very low signals. In this paper we present a facile procedure applicable to whole sets of EFTEM maps acquired on a sample that is evolving over time. The main aim is to find a common method to treat the images features, in order to make them as comparable as possible without affecting the information there contained.
\end{abstract}




\section{Introduction}

Energy Filtered Transmission Electron Microscopy (EFTEM) is a well-known tool for obtaining easily filtered images or elemental maps. It combines the advantages of parallel TEM imaging, i.e. the spatially resolved scattering of a parallel electron beam, with the electron energy loss spectroscopy (EELS). In this way, the inelastic scattered electrons of the incident beam, that lost energy during the interactions with the atomic electron shells of the elements constituting the investigated specimen, can be selected accordingly to the ionization edge of a certain element and used to yield its spatial distribution, which is commonly indicated as elemental map (Egerton, 1996).

In the twenty years from its establishment, many studies have been devoted towards the improvement of the technique for both qualitative and quantitative purposes, albeit other techniques such as spatially resolved EEL and EDX spectroscopy have since emerged as quantification tools of choice (Verbeeck et al., 2004). A part of them focused on the means to fine-tune the acquisition parameters of the elemental maps in order to boost the signal, while others focused on the enhancement of the signal-to-noise ratio (SNR) by acting on the noise, either by subtracting the background contributions through subsequent approaches (going from the jump-ratio to the three-window, and to the multi-window method) or by statistical approaches such as multivariate statistical analysis (Heil et al., 2012; Lozano-Perez et al., 2009; Moore at al., 2002). However, albeit different, these approaches were addressed mainly to improve the SNR of single element maps, such as the automatic brightness and contrast adjustment performed by dedicated EFTEM software, at the end of each acquisition. These automatic processes depend on the single image's features and, when applied to a generic EFTEM dataset, enhance the SNR of each single elemental map independently, with a consequent discrimination between signal and background noise varying map-by-map. Besides, when the signal/noise discrimination is not automatized but user-tuned, the risk of biasing due to the different subjective perception of experimental data cannot be ruled out and can significantly affect the discrimination between what is "signal" and what is "background". As a consequence, a possible undesirable effect of human-induced biasing can be strongly increased when the EFTEM dataset contains low SNR chemical maps. Here, the "naked eye" discrimination between minimum level of signal and maximum level of noise becomes blurry and can generate humaninduced "artifacts", resulting in an strengthening of major features and a loss of fine and weak details in the element maps.

In the present work, we propose a facile method of noise filtering based on a a priori defined approach, suitable for any generic set of EFTEM maps and using open source image treatment software, such as ImageJ (Schneider et al., 2012). This method aims at minimizing the human-based biasing effects through a correct choice of the best signal-to-noise condition taking into account the whole brightness and contrast range on a reference map and subsequently applying this correction to all filtered images of a given EFTEM dataset. Although not suitable for eliminating systematic sources of error from experimental data, this approach allows to easily compare different maps constituting a set of EFTEM data acquired on materials 
subjected to compositional changes over time, while keeping the whole spectral information totally preserved.

\section{Materials and Methods}

Energy Filtered TEM (EFTEM) analysis was performed with a JEOL JEM-2200FS microscope, equipped with a field emission gun working at an accelerating voltage of $200 \mathrm{kV}$, a CEOS spherical aberration corrector of the objective lens allowing to get a spatial resolution of $1 \AA$, and an in column Omega energy filter, using a contrast aperture of about $10 \mathrm{mrad}$ to reduce mostly chromatic aberration. EFTEM maps were acquired according to the three-window method (two pre-edge and one postedge window) for background subtraction. Elastic (zero-loss) images with a 10-eVwide energy slit were acquired as reference. The EFTEM images were acquired by a Gatan 2kx2k US1000 CCD camera (Gatan Inc., Pleasanton, CA, USA) and the relevant files saved in the DM3 Gatan format.

Signal/noise treatment operations were performed using Image for the analysis and manipulation tasks and a spreadsheet was used for the relevant calculations.

\section{Results and Discussion}

The image treatment approach here reported is based on a three-step process: i) binning, ii) normalization and iii) referencing of experimental EFTEM maps, and requires some common sense prerequisites to be satisfied for its proper working. The basic point that we will assume is that the whole imaging dataset to be treated should have been acquired during a single session, so that any possible external source of error, such as electron density current variation, microscope alignment, image detector's dark and gain references and substrate thickness, can be effectively considered as systematic and left aside as fixed parameters with regards to each individual set.

Since the TEM column is now considered as a closed system under high vacuum, no additional matter can be created and appear on the TEM grid during the data acquisition. On the other hand, phenomena such as phase changes, coalescence or migration involve the motion of atomic species on the TEM grid or thin support, while background contributions should be hypothetically related to the sole TEM grid. A crucial point is that the whole grid, and particularly the observed samples, should be as clean as possible, i.e. with no trace of sources of signal that could act as an aspecific and sample-related source of chemical information. This means that beam-sensitive samples should be analyzed with the utmost care and that conservative acquisition parameters should be identified in advance to the actual experiment, in order to guarantee the integrity of the sample. On the other hand, an insufficient cleaning will affect negatively the data acquisition in different and not predictable ways, thus compromising the interpretation and overall quality of the experimental data. For instance, in studying the time evolution of materials obtained by chemical synthesis, the possible presence of unreacted precursors and/or of 
residuals of capping agents on the substrate where the sample is deposited is likely to mask the proper signal to be mapped, possibly giving spatially aspecific information and leading to variations in the level of background coming from different substrate zones (uneven quantities of precursors/residual will lead to different response from the sample in different zones of the substrate), finally resulting in covering the signal coming from the sample parts that are actually changing composition over time.

Our signal/noise treatment is here expressed as an algorithm flowchart and will first be described as a walkthrough in order to clarify the actions taken and the programs used in each step (Figure 1). A direct application of this image treatment protocol on experimental EFTEM dataset is then presented as an example/tutorial. Prior to starting the treatment reported in the following, the EFTEM maps have to be evenly dimensioned and put at full-contrast limit, in order to easily verify their correct alignment and lack of artifacts.

A TEM/EFTEM image can be considered as a matrix of pixels, with each pixel displaying a specific grey level. Since the full contrast range of images in the DM3 format is specific to each single image, the maps should be converted to 16-bit, so that the full contrast range is common to the whole set. In this direction, the histogram of grey levels will be distributed over 65536 channels, from white (0channel) to black (65535-channel), according to the ImageJ software's notation, and the total pixel count of grey scale distribution will be a integer number, which depends on the pixel size of the starting image $(1024 \times 1024,2048 \times 2048$ or more). The total pixel count (area of distribution) is then constant for every image and independent from the adopted dynamical range, i.e. 8-bit or 16-bit, used to express the grey-scale. Moreover, the grey scale distribution - grey scale histogram remains unchanged in terms of curve trend, as position of maxima, minima and inflection points, because of it is completely determined and included within the fixed limits white (origin) and black (end), and it is a function directly correlated to the features recorded in the image itself. The binning operation performed by ImageJ (through the Image/Type/ 16-bit menu) converts the generic contrast range of a RGB image to a 16-bit image with 256-channels, each comprising 256 grey levels for a total of $65536\left(2^{16}\right)$ levels, where channel 0 corresponds to white and channel 255 to black. By calculating the histogram of brightness and contrast for each map (through the Analyze/Histogram ImageJ, menu), the distribution of grey levels can be analyzed and its mode, minimum (Min) and maximum (Max) can be retrieved. Each map is characterized by its proper brightness and contrast histogram, meaning that variations in the parameters of mode, Max and Min will result in variations in the levels of grey scale composing each actual image.

Consider a set of maps taken in a single EFTEM session with the same acquisition conditions (i.e. exposition time, beam concentration, position and width of the filtering window). The brightness and contrast normalization process aims to uniform the grey levels in all images in order to have the each $\mathrm{i}^{\text {th }}$-channel corresponding to the same shade of grey in all the images of the set. This goal can be achieved by a two-step process of reference finding and normalization: i) all the 
histograms must be analyzed in order to find the smallest mode (mode $e_{\min }$ ); ii) the grey-levels histogram of each image must be shifted so that its mode is superimposed to mode min. $_{\text {. }}$

The first step is intended to find the map with maximized visibility, because in the grey scale histogram the lower the $\mathrm{i}^{\text {th }}$-channel is, the more the corresponding greycolored pixels tend towards white. The mode was preferred to the "mean" or "median" values as a reference point because an unambiguous "reference point" was needed for each histogram to perform the referencing and normalization steps. Since histograms obtained by the greyscale levels cannot be granted to be symmetrical distributions, choosing any mean value as reference point would have resulted in imposing the superposition of a series of greyscale channels that are differently positioned inside each histogram, depending on its symmetry and width. The second step is the actual normalization, because the "shift" of the maps in terms of grey levels along the white-to-black 256 channels axis towards the common minimum value mode $e_{\min }$ leads to the superposition of the histograms in terms of grey levels. This guarantees the uniformity of the image maps in terms of the channel-to-grey level equivalence and the maximization of their signal, because the best-case scenario available in the dataset is being used as common reference.

This normalization task can be performed in ImageJ by selecting a map, choosing from the top menu Process/Math/Subtract and inserting a value of choice $D_{i}$, defined as

$D_{i}=$ mode $_{i}-$ mode $_{\min }$

After this normalization procedure has been completed for every $\mathrm{i}^{\text {th }}$ image, the greylevels are uniformed through the whole dataset.

The next step is intended to maximize the SNR in the maps. First, a reference map must be chosen among those of the dataset, then the maximization of the SNR must be achieved in the reference map. Both operations should be faced with proper consideration, because selecting an improper reference map and/or performing an incorrect SNR maximization would cascade through the whole dataset. In fact, the process of choosing the reference map should not be limited to finding the "best looking" image, but it must take into account the kind of experiment performed during the acquisition of the data. Since the data acquired through a generic dataset is likely to show some sort of variation of the sample, a proper reference map should be the one showing as clearly as possible the features of the sample before the experiment takes place. This means that the image chosen as reference will be the first one in a time series or the starting image in a in situ annealing experiment, because in those conditions the proper signal should be expected only in correspondence to the actual sample as shown by Zero Loss TEM images. Conversely, choosing a generic map where the difference between background and proper signal is the clearest as the reference can be hazardous, because referencing all the maps to a generic intermediate step of the experiment could lead to an improper SNR assessment. 
The actual SNR maximization process requires the choice of a band-pass window within the grey-levels histogram of the reference map. This task can be performed by manually adjusting the "Minimum" and "Maximum" channels of interest (from the Image J menu Image/Adjust/Brightness \& Contrast), in order to remove the background noise (position of "Minimum") and improve the signal intensity (position of "Maximum"). In particular, the "Minimum" channel should be the minimum value that eliminates the background signal from the regions of the reference map where no sample is present without affecting the proper signal in the regions of the map where the sample is present. A lower-than-necessary "Minimum" channel won't completely remove the background signal, while a higher-thannecessary value will remove the background along with part of the proper signal, thus altering some features of sample. The "Maximum" channel on the other hand should be the one that maximizes the intensity of the proper signal without altering the main features of the sample recorded in the map. A too low "Maximum" value will affect features such as size and shape of the sample in the map, while a too high value will result in a less visible and greyish proper signal. The whole process of choosing the band-pass window can be thought as a sort of further binarization, where the chosen "Minimum" and "Maximum" channels set the level of black and white, respectively, and the channels in-between function as intermediate grey levels.

Once the background noise has been minimized and the proper signal has been maximized in the reference map, the position and width of the band-pass window must be quantified in the reference map and their equivalents must be calculated for the other maps in the normalized dataset.

In the reference map the width of the band-pass window can be obtained as

$$
B W_{R E F}=\text { "Maximum" }_{R E F}-\text { "Minimum" }_{R E F}
$$

where "Maximum" and "Minimum" indicate the channel numbers in the Brightness \& Contrast menu of ImageJ; the position of the band-pass window can be put in relation to the mode of the distribution of grey-level channels as

$$
X_{R E F}=" \text { Minimum" }_{R E F}-\text { mode }_{R E F}
$$

Given a generic map of a normalized dataset and its corresponding grey-levels histogram, there must be a fixed proportionality between the width of the greylevels histogram and the width of the band-pass window. This fixed proportionality goes through the definition of the interval $I=$ Max-mode in the grey-levels histogram of each map of the dataset, so that the band-pass window width can be defined as

$$
B W_{i}=I_{i} \frac{B W_{R E F}}{I_{R E F}}
$$


Once the band-pass window widths have been set, their position in the corresponding grey-level histograms can be calculated from the relation

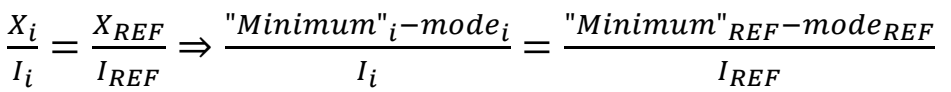

Where $X_{i}=$ "Minimum" $_{i}-$ mode $_{i}$ indicates the position of the generic $\mathrm{i}^{\text {th }}$ band-pass window in the same fashion as that defined in equation (2).

This leads for each map to define a "Minimum" ${ }_{i}$ and a "Maximum" ${ }_{i}$ as follows:

"Minimum" $_{i}=\operatorname{mode}_{i}+X_{i}=\operatorname{mode}_{i}+\frac{I_{i}}{I_{R E F}} \cdot\left(\right.$ "Minimum" $\left._{R E F}-\operatorname{mode}_{R E F}\right)$

Maximum" $_{i}=B W_{i}+$ Minimum $_{i}$

These values can subsequently be used in the Brightness \& Contrast menu of ImageJ to select the desired channels and obtain the final treated maps.

In order to better clarify the actual steps involved in our approach of postacquisition a priori image treatment, a set of previously published EFTEM elemental maps has been treated according to our algorithm, providing an example of the advantages of this method over the standard automated approach. In particular we are going to treat the copper maps recorded during an in situ annealing experiment showing a pervasive temperature-triggered chemical and structural transformation from $\mathrm{CdSe} / \mathrm{Cu}_{3} \mathrm{P} / \mathrm{CdSe}$ heterostructures to $\mathrm{Cu}_{2} \mathrm{Se}$ nanocrystals (De Trizio et al., 2013).

The first step requires the preparation and conversion of the maps to a more suitable format. The images are first set to full contrast under Digital Micrograph, while Image J can be used for removing the peripheral/frame artifacts generated from the automated three windows method for background subtraction and for converting the RGB-type elemental maps into 16-bit tiff images (Figure 2, top, A-C). The conversion from DM3 Gatan format to 16-bit tiff causes the binning of the image-specific channels constituting the full contrast histogram to a final common set of 256 channels (Figure 2, bottom).

The subsequent step demands the normalization of the greyscale used to display the signal in the images of the EFTEM dataset. The greyscale histograms obtained from the binned maps must be analyzed and their parameters compared in order to determine which one displays the lowest value of mode (Figure 3, left). All the histograms are then shifted along the greyscale axis, so their modes are superimposed to the minimum one, resulting in a variation of the greyscale in all the images that enhances the signal to the best case scenario of the dataset (Figure 3, right).

Once the greyscale in the maps has been normalized, the actual band-pass window can be applied on a reference elemental map of choice. As previously stated, the choice of this reference map should be related to the kind of experiment recorded in the dataset. Since in the present case we are referring to an in situ annealing 
experiment, the logical choice for a "safe" reference is the copper map recorded at room temperature, before any actual change has taken place (Figure 4A). The actual treatment of the reference map consists in the manual selection of an interval of channels in its greyscale histogram: first the "Minimum", which is the smallest channel that minimizes the background noise signal, hence the "Maximum" which is intended to maximize the remaining signal without loosing information (Figure 4B). The band-pass window, indicated in cyan, can then be defined in width and position with respect to the mode of the histogram according to equations (1) and (2).

The last step combines the previous results by applying the treatment operated on the reference map to the whole dataset of normalized maps. The equivalent width and position of the band-pass windows for each element map can be obtained by equations (3) and (4), so that the corresponding "Minimum" and "Maximum" channels can be assessed according to equations (5) and (6). Thus, the normalized maps are obtained by selecting the calculated band-pass windows, again indicated in cyan, in the corresponding greyscale histograms (Figure 5).

A direct comparison between the advantages of our a priori method and the "standard" procedure (that is, the independent assessment of the brightness and contrast levels for each elemental map of the dataset) can be appreciated in Figure 6 , where the original set of element maps is presented side to side with the ones treated following our approach. As a first remark, the intensities of the $\mathrm{Cu}$ signal are now consistent with the evolution of the sample caused by the annealing: while the average intensity in the unfiltered maps varies randomly with the increasing temperature, the filtered maps feature a steadier intensity through the whole set, whose variations are consistent with the $\mathrm{Cu}$ diffusion and phase transformation processes. Moreover, in the "standard-treated" maps the feeble signal of $\mathrm{Cu}$ outdiffusing from the $\mathrm{Cu}_{3} \mathrm{P}$ domains of the heterostructures is lost, because it's totally masked by the background noise. Conversely, the same $\mathrm{Cu}$ maps treated according to our a priori method reveal a weak but clear $\mathrm{Cu}$ signal between and around the nanoparticles, already at $200^{\circ} \mathrm{C}$. This feeble $\mathrm{Cu}$ signal, resulting from the filtering of background, can now be directly correlated to the thermal out-diffusion process. Here, the possibility to discriminate $\mathrm{Cu}$ signals, even though feeble, in such a thermal-activated diffusion process of atomic species can give rise to new insights into the mechanisms governing the reactions involving the chemical and structural transformations at nano-scale.

\section{Conclusions}

The post acquisition image treatment we presented here takes advantage of the common features of a dataset of EFTEM maps to assess a common adaptive signal/noise threshold valid for each map. On the one hand this approach aims to reduce the possible formation of human-induced artifacts due to naked-eye interpretation of the maps, while on the other hand enhancing the visibility of faint elemental signals by maximizing the SNR and improving the consistency of the signal/noise discrimination within the whole dataset. 


\section{References}

De Trizio L, De Donato F, Casu A, Genovese A, Falqui A, et al. 2013. Colloidal $\mathrm{CdSe} / \mathrm{Cu}_{3} \mathrm{P} / \mathrm{CdSe}$ nanocrystal heterostructures and their evolution upon thermal annealing. Acs Nano 7: 3997-4005.

Egerton RF. 2011. Electron Energy Loss Spectroscopy in the Electron Microscope, $3^{\text {rd }}$ edition. New York: Plenum Press. 431p.

Heil T, Gralla B, Epping M, Kohl H. 2012. Improving the reliability of the background extrapolation in transmission electron microscopy elemental maps by using three pre-edge windows. Ultramicroscopy 118: 11-16.

Lozano-Perez S., de Castro Bernal V, Nicholls RJ. 2009. Achieving sub-nanometre particle mapping with energy-filtered TEM. Ultramicroscopy 109:1217-1228.

Moore KT, Stach EA, Howe JM, Elbert DC, Veblen DR. 2002. A tilting procedure to enhance compositional contrast and reduce residual diffraction contrast in energyfiltered TEM imaging of planar interfaces. Micron 33:39-51.

Schneider CA, Rasband WS, Eliceiri KW. 2012. NIH Image to ImageJ: 25 years of image analysis. Nat. Methods 9: 671-675.

Verbeeck J, Van Dyck D, Van Tendeloo G. 2004. Energy-filtered transmission electron microscopy: an overview. Spectrochim. Acta B 59: 1529-1534. 
Figures

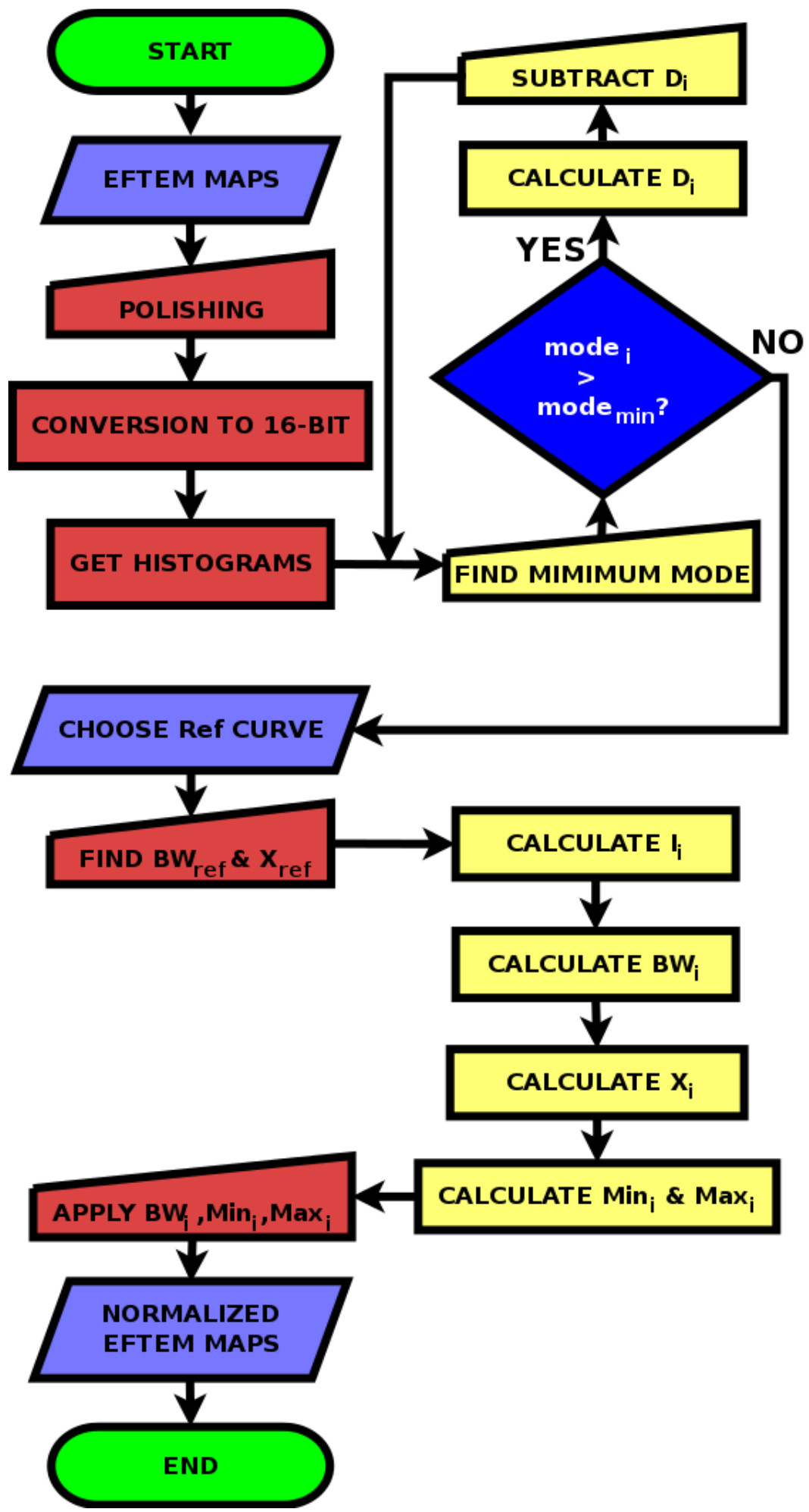

Figure 1 

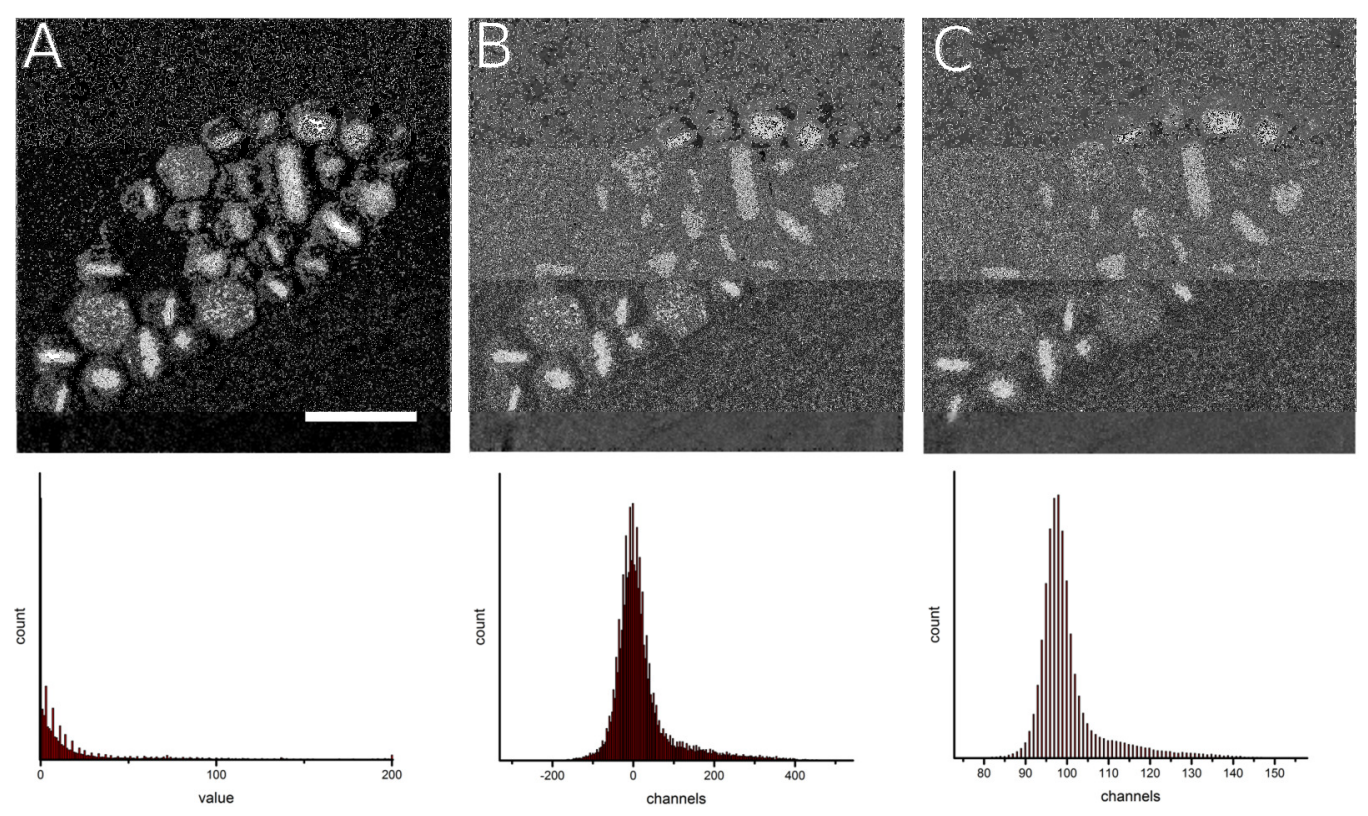

Figure2 

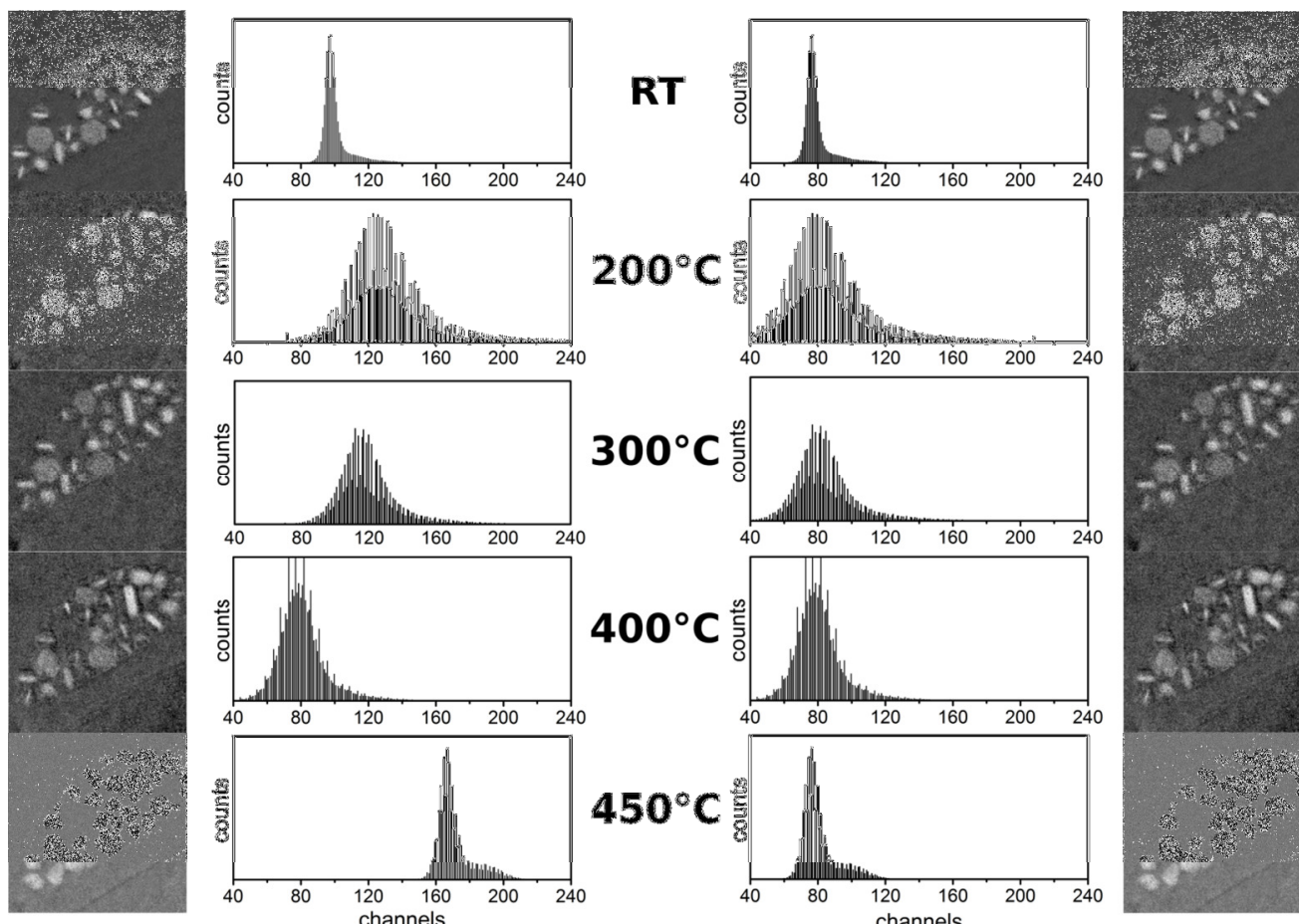

PRE TREATMENT

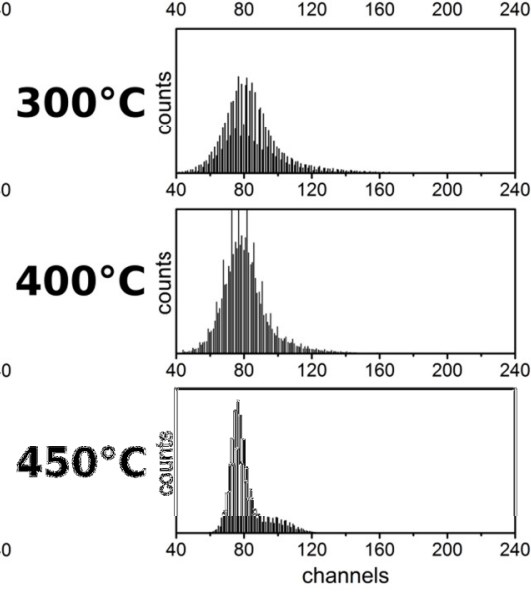

POST TREATMENT

Figure 3 

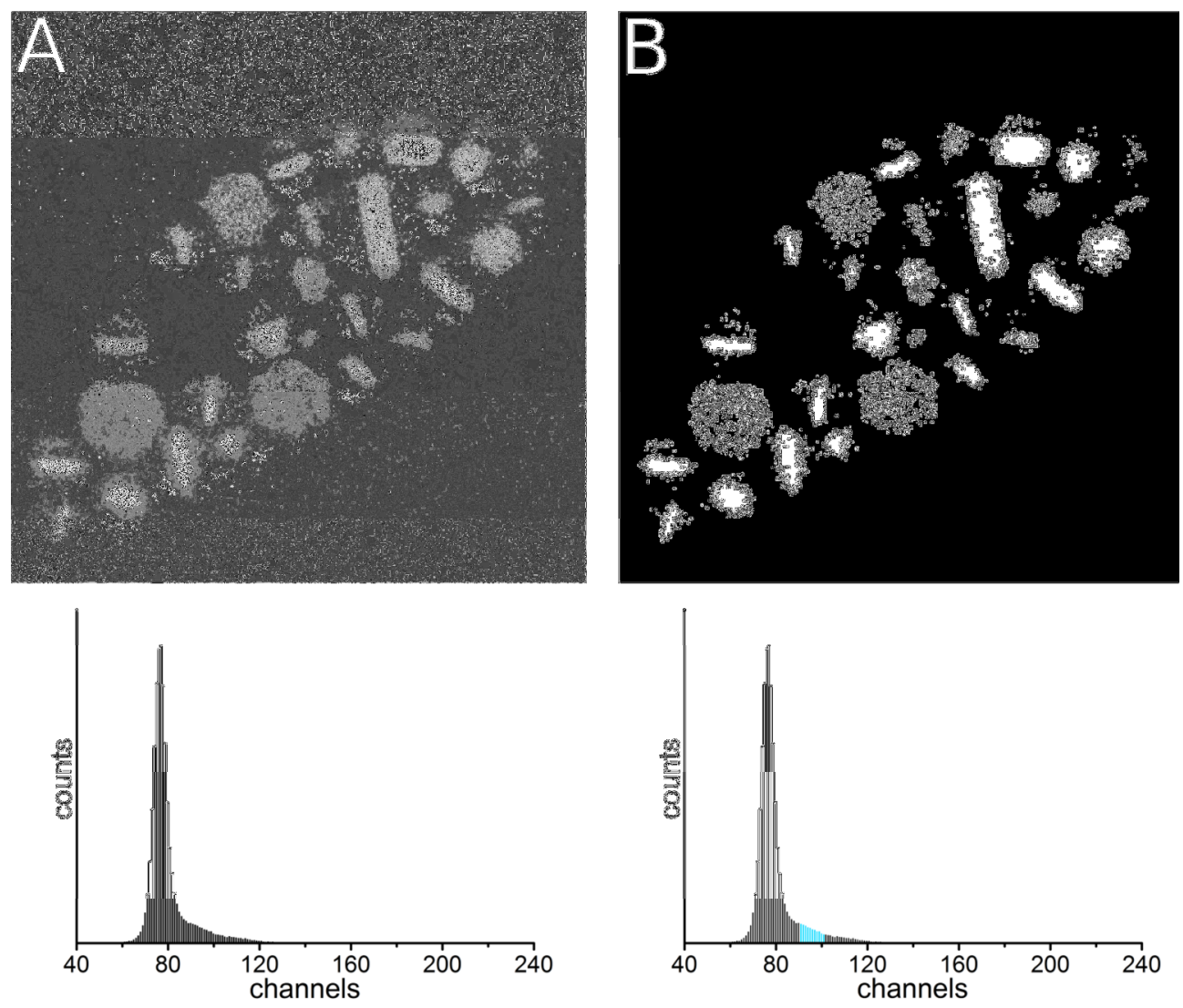

Figure 4 

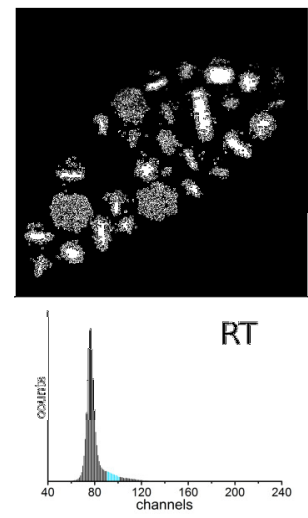
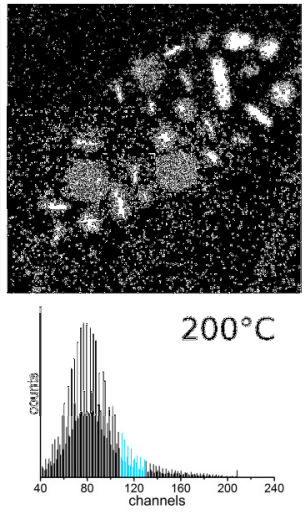

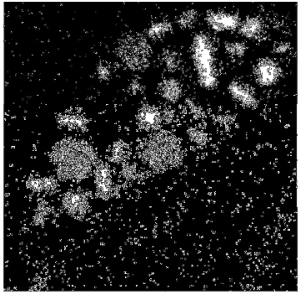

$300^{\circ} \mathrm{C}$

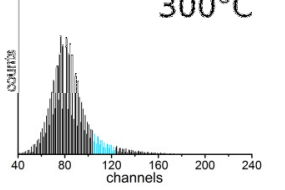

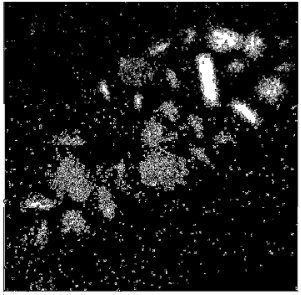

$400^{\circ} \mathrm{C}$

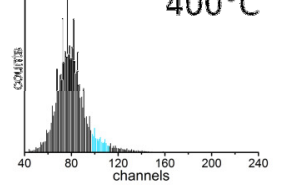

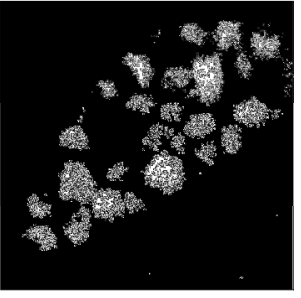

$450^{\circ} \mathrm{C}$

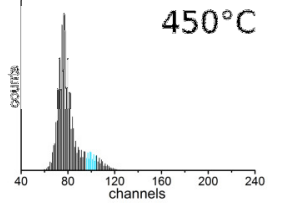

Figure 5 

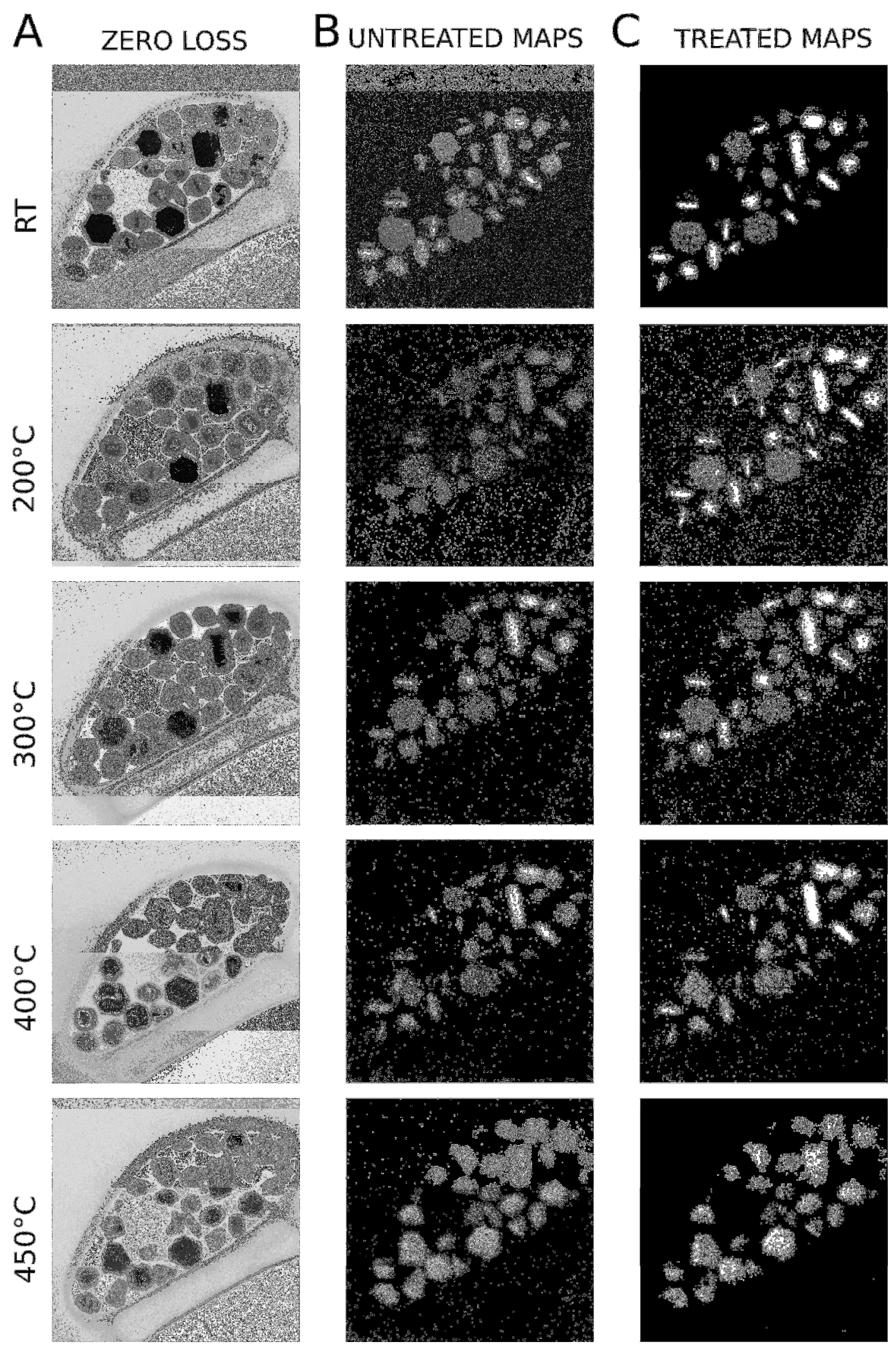

Figure 6 


\section{Figure legends}

Figure 1: Flow chart depicting the algorithm of the image treatment process. Redfilled blocks indicate ImageJ-based actions and yellow-filled block indicate spreadsheet-based actions. Purple-filled blocks indicate user-defined input/output.

Figure 2: Preparation and binning of an automatically balanced Cu EFTEM map in DM3 format (A). The map is set to the full-contrast limit (B) and converted to 16-bit format (C). The corresponding histograms show which channels of the greyscale histograms are used for the formation of each image, going first from a portion of the greyscale histogram $(\mathrm{A})$ to the full greyscale histogram of image-specific levels of the DM3 format (B), then to the absolute 256 channels of the 16-bit format (C). The scalebar is $100 \mathrm{~nm}$.

Figure 3: Normalization of a set of EFTEM maps. The minimum mode is identified among the histograms of the untreated maps (left) and the normalization of the greyscale levels determines the alignment of the modes of all the greyscale histograms (right).

Figure 4: Identification of the band-pass window of choice in the reference map. The choice of Minimum and Maximum levels in the greyscale histogram of the full contrast map (A) determines a binarization of the map formed using the channels in the band-pass window (B). The channels selected by the band-pass window are indicated in cyan.

Figure 5: Treated normalized elemental maps (top) and corresponding greyscale histograms (bottom). The channels selected by each band-pass window are indicated in cyan.

Figure 6: Comparison between treated and untreated elemental maps. Zero loss images (A) can be used as visual reference for the set of automatically and independently balanced elemental maps (B) and those treated following our approach (C). 